\title{
The Quantiles Estimation in the Monsoon Season of Seven Unguaged Sites of Punjab Pakistan with Regional Rainfall Frequency Analysis
}

\author{
Uzma Nawaz*, Tooba Nihal, Aisha Ejaz
}

\begin{abstract}
Pakistan is located between the latitude $25^{\circ}$ and $36^{\circ}$ with strong spells of monsoon rains showering from June to September with somewhat variation according to location. Heavy rains in monsoon season make dams, rivers and lakes overflow causing destructive floods. The scenario provoked the study on the estimation of regional rainfall quantiles of Annual Maximum Monsoon Rainfall Totals (AMMRT) of the seven unguaged sites of the Punjab province in Pakistan. The estimates are obtained from L-moment based Regional Rainfall Frequency Analysis (RRFA). The monsoon data series of the seven sites were found random and identically distributed with no serial correlation. These results were the output of the run test, lag-1 correlation and Mann-Whitney $U$ test. The $L$ moment based discordancy measure exposed no site discordant in the group of seven. The heterogeneity measure concluded a homogenous region of the seven sites. The L-moment Ratio diagrams exposed the Generalized Normal as the best choice of the regional distribution and is used for the quantile estimation. The estimates of the study may be used for the estimation of the rainfall quantiles of the seven sites for different return periods. The estimates will be provocative to design future preventive measures for the harmful impact of hydro meteorological events at these sites in Punjab Pakistan.
\end{abstract}

Keywords - L- moments, Regional Rainfall Frequency Analysis, Linear Models, Discordancy Measures, Heterogeneity Measure. 